\title{
Fluxon electronic devices
}

\section{Pedersen, Niels Falsig}

\section{Published in:}

IEEE Transactions on Magnetics

Link to article, DOI:

10.1109/20.133925

Publication date:

1991

\section{Document Version}

Publisher's PDF, also known as Version of record

Link back to DTU Orbit

Citation (APA):

Pedersen, N. F. (1991). Fluxon electronic devices. IEEE Transactions on Magnetics, 27(2, 4), 3328-3334. https://doi.org/10.1109/20.133925

\section{General rights}

Copyright and moral rights for the publications made accessible in the public portal are retained by the authors and/or other copyright owners and it is a condition of accessing publications that users recognise and abide by the legal requirements associated with these rights.

- Users may download and print one copy of any publication from the public portal for the purpose of private study or research.

- You may not further distribute the material or use it for any profit-making activity or commercial gain

- You may freely distribute the URL identifying the publication in the public portal

If you believe that this document breaches copyright please contact us providing details, and we will remove access to the work immediately and investigate your claim 
FLUXON ELECTRONIC DEVICES

\author{
N. F. Pedersen \\ Physics Laboratory I \\ The Technical University of Denmark \\ DK-2800 Lyngby, Denmark
}

\section{Abstract}

The fundamental properties of fluxons on Josephson transmission lines are discussed from both a theoretical and an experimental point of view. Various applications within superconducting analog and digital electronics are evaluated.

\section{Introduction}

Fluxons on long Josephson junctions have very unusual and interesting properties.

The possible applications of those unique properties have stimulated a lot of recent research activity. The present paper focusses on that topic and discusses the potential applications within superconducting electronics. Before addressing that topic, however, we devote a section to remind the reader of the basic theory and properties of fluxons on the Josephson transmission line (JTL). This section is followed by a section that deals with basic experimental properties illustrated by examples from the published literature. In the sections thereafter digital applications, $r f$ applications and analog amplifier applications based on fluxons are discussed. The paper is concluded by a summary.

\section{Basic Theoretical outline}

A fluxon is a quantum of magnetic flux that has solitonic properties and may exist for example on a Josephson transmission line (JTL). Here it will appear as a localized $2 \pi$ phaseshift, that may move much like a particle under the influence of driving forces (e.g. bias current) and damping. It has a vortex current structure with a corresponding magnetic field in the $\mathrm{pl}$ ane of the barrier and at right angles to the length of the JTL.

The associated magnetic flux is quantized in units of the flux quantum $\Phi_{0}=2.07 \times 10^{-15} \mathrm{Vs}$.

A7though a full derivation cannot be given, a few of the basic properties will be out1ined here. (A more complete description may be found in for example refs. $(1,2))$. For an extended (in the $x$ direction) one dimensional Josephson junction, the dynamics of the quantum mechanical phase difference between the two sides is described by the following partial differential equation 1,2 , which is

Manuscript received September 24, 1990. referred to in the literature as the perturbed sine-Gordon equation

$$
-\phi_{x x}+\phi_{t t}+\alpha \phi_{t}+\sin \phi=\eta
$$

This equation is obtained by adding a spatial term - $\phi_{x x}$ to the usual differential equation for a small Josephson junction. In Eq. (1), $\phi(x, t)$ is the quantum mechanical phase difference, $\alpha=1 / \sqrt{\beta_{c}}$ is the damping parameter ( $\beta C$ is the usual McCumber parameter given by $\beta C=$ 2e $R^{2} I_{0} \mathrm{C} / h$, where $I_{O}, \mathrm{C}$, and $\mathrm{R}$ are the supercurrent, capacitance and shunt resistance per unit area respectively). $\eta$ is the dc bias current normalized to $I_{0}$. Time $t$ is normalized to $1 / \omega_{0}$ where $\omega_{0}=\sqrt{2 e l_{0} / h C}$ is the Josephson plasma frequency, and the spatial dimension $x$ is normalized to the Josephson penetration depth $\lambda_{J}=\sqrt{h / 2 e d \mu_{0} J_{0}}$, where $d=2 \lambda_{l}+t$ is the magnetic thickness of the barrier, and $J_{0}$ is the supercurrent density.



Fig. 1 Geometrical configurations of the JTL. (a) overlap junction, (b) inline junction, (c) annular junction.

The solution to Eq. (1) depends on the geometry of the junction we consider. In Fig.l various possibilities are shown. The simplest case is the infinite line (no boundary conditions) of the overlap geometry (Fig. la) or the annular junction (Fig. 1c) of length $1>>1$, where we can use periodic boundary conditions. In both cases we may consider $\eta$ spatially independent and assume a travelling wave solution $\phi(x, t)=\phi(x)$; here $x=x-u t$, where $u$ is the velocity normalized to the speed of light in the barrier $\bar{c}=c \sqrt{t / d \epsilon}$, c being the vacuum speed of light and $\epsilon$ the relative dielectric constant, $(u<1)$.

The traveling wave solution to Eq.l may be found by perturbation analysis 3,2 or in a particularly simple way as described $\mathrm{in}^{4}$. The result is, that the velocity is determined by a power balance between the bias $\eta$ and the losses given by $\alpha$ i.e.

0018-9464/91/0300-3328\$01.00 (c) 1991 IEEE 


$$
u=1 / \sqrt{1+(4 \alpha / \pi \eta)^{2}}
$$

and the voltage $v$ is given by

$$
v=2 \pi(u / 1)
$$

for $1 \gg 1$. A typical current voltage characteristic, Eq. $(2,3)$, is shown in Fig. 2a. This curve is often in the literature referred to as a zero field step. The shape of the soliton is approximately given by $2-4$

$$
\phi^{(s)}=4 \tan ^{-1}\left(e^{\xi}\right)
$$

Here $\xi=(x-u t) \gamma(u)$, where $y(u)=1 / \sqrt{1-u^{2}}$ is the Lorentz factor. The shape of the phase difference $\phi^{(s)}$ together with the junction voltage $\phi_{t}^{(s)}$ (or the vortex current $\left.\phi_{x}^{(s)}\right)$ is qualitatively shown in Fig. 2b. The voltage has the shape of a pulse; it narrows and increases in amplitude as the velocity approaches 1 (speed of 1 ight)
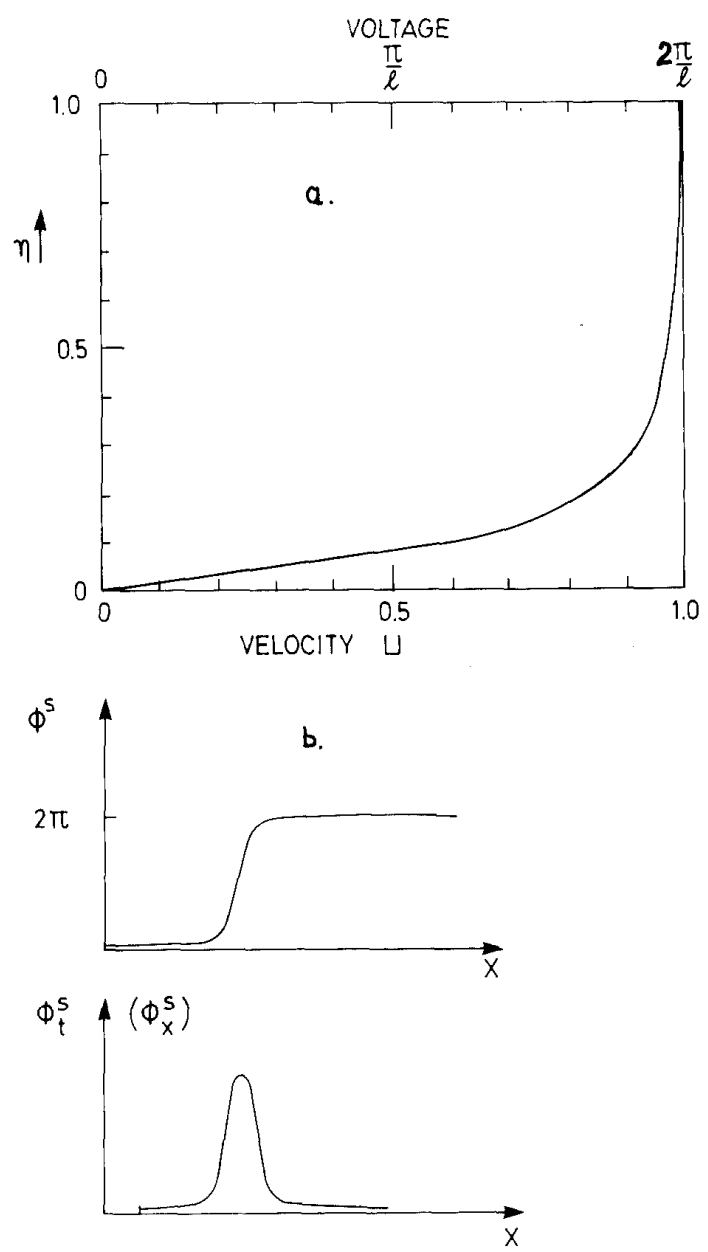

Fig. 2 (a). Soliton velocity $u$ and voltage $v$ versus bias $\eta$ with $\alpha=0.1$. (b). Qualitative drawing of soliton $\phi^{s}$ and its time derivative $\phi_{t}^{s}$ (spatial derivative $\phi_{x}^{s}$ ). because of the Lorentz contraction built into Eq. (4). Hence the properties of the fluxon on the JTL may also be described as those of a relativistic particle. Eqs. $(2,3)$ break down, if the line is not very long, so that boundary effects play a role. Eqs.2-4 and the Lorentz invariance break down, if losses $(\alpha)$ are large and/or $\eta$ approaches 1 , i.e. the perturbations become large. Corrections can be worked out $2-4,5$, but will not be dealt with here.

The simplest effect of a boundary at $x=1$ is the obvious that no currents can pass beyond $x=1$. This gives the boundary condition

$$
\phi_{x}(l, t)=0
$$

Typically - though not always - this boundary condition implies that a fluxon is reflected (elastically) as an antifluxon with the variable $\xi=(x+u t) \gamma(u)$ to be inserted in Eq. 4, i.e. $u$ and $\phi_{x}$ changes $\operatorname{sign}^{2}, 3$.

More elaborate schemes for fluxon electronics require the simul taneous presence of both fluxons and antifluxons. Fluxons and antifluxons have interesting and unusual properties; for example they may pass through each other in a collision - provided the bias current exceeds a threshold bias current $\eta_{t h}$ given approximately by 6

$$
\eta_{t h}=(2 \alpha)^{3 / 2}
$$

For bias below $\eta_{t h}$ the fluxon and antifluxon annihilate each other, and the energy is dissipated as small oscillations on the JTL. Fig. 3 shows a numerical calculation of a fluxon-antifluxon collision 7 with $\eta>\eta_{t h}$.

Solitons in the sense discussed here apply to other physical systems such as a parallel array of microbridges 8 , charge density waves $^{9}$ and a pendu 7 um chain 10 . For i1lustration Fig 4 shows a (computed) annular pendulum chain, which is a mechanical analog of an annular junction (Fig IC) containing one fluxonl1.



Fig. 3 Collision between a soliton and an antisoliton with $\alpha=0.2$. $\eta=0.22$, normalized length $l=40$. The wiggles for small time are transients that have not yet been damped out. (Ref. 7). 


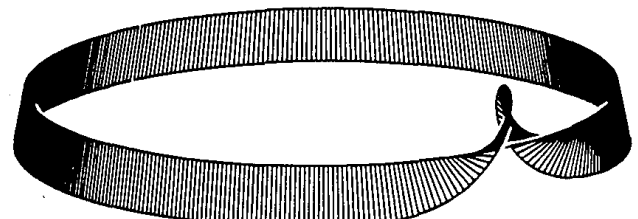

Fig. 4 Computer-generated equivalent pendulum array with a 2J kink. $\alpha=0.02, \eta=0.4$, and $l=8$ (after Ref. 11).

\section{Dynamics on the JTL: Basic Experiments}

There are several ways to experimentally observe the the phenomena discussed above. The simplest experiment to perform with fluxons on the JTL is to measure its IV-curve. Fig. 5 shows an IV curve measured for an annular $J T L 11$. The numbering on the curves correspond to the number of fluxons and antifluxons. Such measurements measure the average velocity of the fluxons c.f. Eq. (3). The first experiment in which fluxon dynamics were described, was such an IV curve measurement of an overlap type of junction 12 . More direct measurements of the shape of the fluxons have recently become possible with the development of Josephson junction sampling circuits (or even traditional electronics with microprocessor data averaging and analysis). Examples of basic studies using these remarkable measurement techniques are found in 13-16. In ${ }^{14}$ there was an experimental observation of a fluxon reflection. Even a direct measurement of a fluxon-antifluxon collision on a JTL (such as the one in Fig. 3) has been measured by a Josephson sampling circuit 15 . In 16 both the annihilation case $\left(\eta<\eta_{n h}\right)$ and the passing through case $\left(\eta>\eta_{\text {th }}\right)$ was observed directly. (Stationary) fluxons have been measured by STM techniques 17 and electron-holographic interferometry 18 .

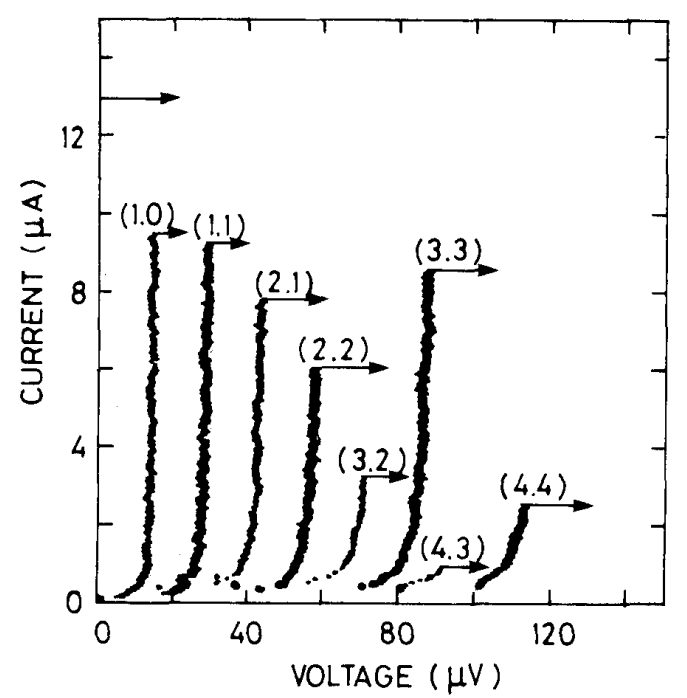

Fig. 5 Experimental I-V curves (superimposed) for the annular junction of Ref. 11. The numbers in paranthesis are the number of participating solitons and antisolitons.
Even in the relatively simple dc - IV - curve measurements 11,19 and in microwave emission measurements 20 can the qualitative behaviour of fluxons together with several details of the dynamics be observed. Methods to move and stop fluxons on the JTL by applying an external control current have been devised, and circuits demonstrating such controlled behaviour have been built and tested 21 . Other experiments, including microwave experiments, will be described in the next sections on applications. Thus to briefly summarize the two first sections, the nature and fundamental dynamical properties of fluxons on the JTL are well understood both theoretically and experimentally.

\section{Digital Applications}

The isolated fluxon was shown to behave like a particle. Therefore the presence or absence of a fluxon in a long junction can represent the two different states in logic operations.

The concept of using a fluxon in digital signal processing was probably first suggested in the early seventies by the group at the Bell Telephone Laboratories 22 . They realized that the socalled flux shuttle had a potential for storing and processing information by treating the fluxon as the fundamental information bit. Their concept of a flux shuttle $e^{22}$, which was also built experimentally23, is still discussed and provides a standard to which newer devices are compared. A flux shuttle is a system of Josephson junctions and inductors as shown schematically in Fig. 6. From the nature and appearence of the equivalent diagram, we see that to realize it, we can imagine both a system consisting of an array of small junctions or al ternatively a spatially modulated JTL. The basic idea is that the position of fluxons on the Josephson transmission line of this special design, can be controlled and manipulated by the application of control currents and magnetic fields. The timescale for switching is determined by the Josephson plasma frequency and may be of order a few picoseconds, while the dissipation per shift22 is of order $10^{-18} \mathrm{~J}$.

An early paper by Nakajima et al. ${ }^{24}$ discussed the design of logic circuits which employed the fluxon as the basic bit of information. The standard logic operations such as OR, AND, and NOT was shown to be possible in networks consisting of Josephson transmission lines alone.

In the days of the IBM Josephson computer project such ideas were also investigated there 25 , although it was not incorporated in the mainstream of the computer project. The associated experiments consisted of a series of measurements on a chain of ten long junctions with

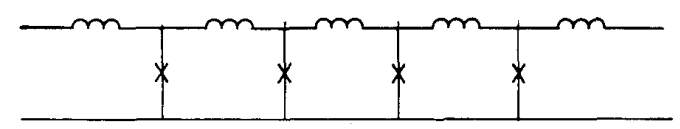

Fig. 6 Schematic drawing of the flux shuttle. 
forks and corresponding control lines. Time delays of order $5 \mathrm{ps}$ and energy dissipation of order $10^{-19} \mathrm{~J}$ per logic operation was projected.

Experimental studies on fluxon digital devices and circuits have been done seriously at Electrotechnical Laboratory (ETL), Tsukuba. At an early stage fluxon transfer devices have been proposed and built, in which external resistors partly connected to long Josephson junctions made fluxon stopping positions. In one case these resistors are placed to shunt the tunnel barriers of long junctions 26 , and in another case they are placed to shunt the inductance of long junctions 21 . Al so a fluxon signal distributor where long Josephson junctions are connected by resistors has been carefuliy tested 27 . The concept of the fluxon transfer devices has been developed to higher sophistication. A fluxon pulser28, and, quite recently, a fluxon shift register 29 has been proposed and demonstrated. The experiments showed the successful 8 bit read-in and read-out operations in a shift register with 16 gates in a long Josephson junction.

Recently Nakajima et al. 30 have proposed a new scheme for a fluxon Josephson computer. The circuits are termed phase mode digital Josephson circuits as opposed to voltage mode Josephson circuits. In the phase mode system a device depends on the existence of many stable states differing from one another by integer multiples of $2 \pi$ in the phase plane. The phase mode system is claimed to be superior in power dissipation to the voltage mode system by two orders of magnitude. The authors 30 realized experimentally several of the common logic operations by elementary circuits composed of SQUIDS and two types of branching points.

\section{R.F. Applications, Microwave Generation.}

In connection with amplifiers and mixers at millimeter and submillimeter frequencies the job of making a local oscillator or pump source is a very important and nontrivial one. At $2-400 \mathrm{GHz}$ traditional sources tend to be bulky and expensive. An important consideration is to have a narrow linewith to avoid excess noise in the amplification process. If one uses Josephson junction mixers and amplifiers, the power requirements are modest - in the range of nanowatts up to microwatts at most. Those requirements can be very well satisfied by fluxon oscillators.

\subsection{Single Soliton Oscillators}

An important reason for using the long Josephson junction oscillator instead of a small junction one is the narrower linewidth of the emitted radiation, but of course also the larger potential power plays a role. A soliton bouncing back and forth in a long junction is perhaps the simplest way of realizing a long Josephson junction local oscillator. In the simplest design 31 a microstrip line is just brought close $(20-50 \mu \mathrm{m})$ to one end of an overlap junction. When the soliton is reflected at the end of the junction, a fraction of the power is coupled to the microstrip line. Since this crude coupling scheme only gives a very weak coupling, it is possible to study the bare fluxon motion by coupling the microstrip line to a conventional microwave receiver at room temperature. This was done for example in ${ }^{20}$, where it was realized, that multisoliton propagation could take place. Such multisoliton motion may keep the fundamental oscillator frequency unchanged, but enhances the power emitted, since more solitons are involved. A typical level of the emitted power at $10 \mathrm{GHz}$, with such a crude coupling circuit as described above, is $10^{-11}-10^{-13}$ Watt. To increase the power more elaborate coupling schemes duch as cavity coupling, slot lines, etc. must be used.

A careful measurement of the emitted linewidth of the radiation was reported in 31 . Again at $10 \mathrm{GHz}$ the 1 inewidth turned out to be of order a few $\mathrm{kHz}$, which is very satisfactory for applications. At $10 \mathrm{GHz}$ many other techniques can be used to produce narrow linewidth oscillators, however the single junction soliton oscillator can easily be scaled to $100 \mathrm{GHz}$, or even 500 $\mathrm{GHz}$, without special technological problems. Theoretically the available power scales at least proportionally to the frequency (possibly even to the square of the frequency) which makes it even more useful at higher frequencies. At $35 \mathrm{GHz}$ output powers of order tens of nanowatts was measured in a single junction soliton generator 32 .

A more elaborate coupling scheme may include the use of a resonant circuit between the long junction and the microstrip. Recently numerical simulations have been performed 33 with the purpose of studying the interaction mechanism between a long junction and a cavity. These simulations demonstrated that it was possible to have a soliton phase lock to the cavity at its resonance frequency. In that process power was delivered to the cavity thereby charging it. With the cavity further coupled to a microstrip circuit the power could be coupled out.

A typical problem in connection with coupling the power out is that the long Josephson junction often has an impedance level below $1 \Omega$ while the microstrip circuit has an impedance level of order $50 \Omega$. Thus either cavity coupling or microstrip impedance transformers or a combination must be used.

For some local oscillator applications (for example some parametric amplifiers) the impedance level of the receiving device is also low. Thus both the local oscillator and the parametric amplifier or mixer may be on the same chip and $50 \Omega$ circuits at microwave frequencies can be avoided altogether. The coupling loss from the long Josephson junction can be reduced considerably. In such an experiment with both the JTL soliton generator and the Josephson junction detector on the same chip, Cirilio et. a1.34 measured about $0.1 \mu \mathrm{W}$ generated at about $75 \mathrm{GHz}$.

It should be kept in mind however, that for many applications, including radio astronomy, the impedance level of incoming signals (antenna) is hundreds of ohms so that somewhere the inconvenient impedance transformation must be made.

A quite different single junction single fluxon oscillator was described by Sakai et al.35. In this remarkable experiment an interrupted annular long Josephson junction with a load resistor in one end and a feedback resistor connecting the two ends was used. 
The oscillation waveform at $24 \mathrm{GHz}$ was measured directly by using a superconducting (Josephson junction) sampling circuit.

\subsection{Coupled (phase-locked) Soliton Oscillators.}

If two identical soliton oscillators can be syncronized to phase-lock, the output voltage doubles and the power increases a factor of 4 . With $\mathrm{N}$ junctions locked in phase the output power would increase as $N$ squared. In a remarkable experiment by the Salerno group 36,37 they used 20 long junctions (of which most of them phase-Tocked) and obtained as much as $0.35 \mu \mathrm{W}$ at $X$-band in a $50 \Omega$ line. This is more than adequate for driving an SIS mixer or a Josephson junction parametric amplifier. Linewidths down to about $5 \mathrm{kHz}$ were measured. The success of the above experiment is remarkable, however the details of the phase-locking is not understood.

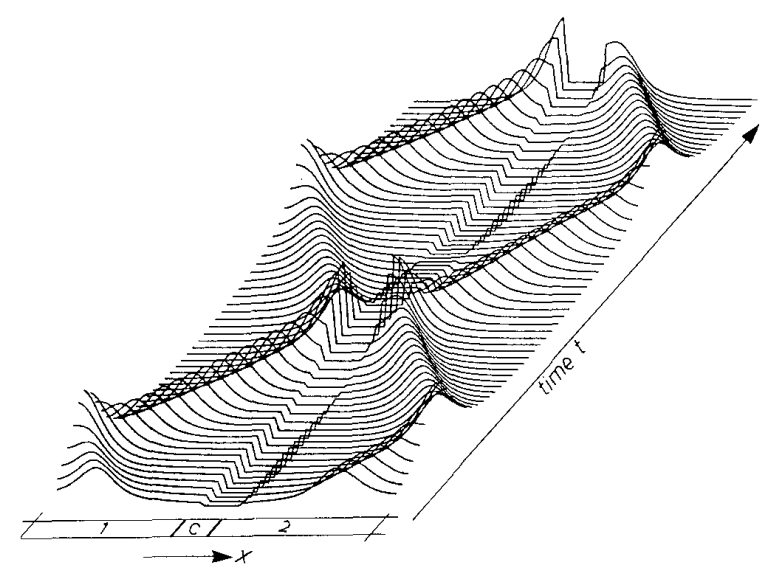

Fig. 7 Two phaselocked long junctions (1 and 2) coupled through cavity (C). $l_{1}=l_{2}=4 . \alpha_{1}=0.05 \cdot \alpha_{2}=0.055$. $\omega_{c}=0.74, Q_{c} \cong 40$ (after Ref. 31).

In order to obtain greater understanding of the fundamentals of the phase-locking process experimental work with a simple system consisting of only two junctions is presently being pursued by the Lyngby group 38 .

Numerically the interaction of two long junctions end-coupled to each other through a common cavity has been studied in 33 . An example of such phase-locking is shown in Fig. 7, which displays quite clearly the syncronisation (with a small phaseshift) of the solitons in the two long junctions, even though the damping parameters were chosen $10 \%$ different in the two lines. The interaction mechanisms between the two junctions and the cavity is quite complicated: in parts of the period current is transferred from a junction to the cavity, and in other parts the current direction is reversed ${ }^{33}$.

Recently Monaco ${ }^{39}$ har performed detailed measurements on a 20 junction array fabricated with almost identical and well coupled long Josephson junctions. The array has the same geometry as in 36,37 . In this work he was able to bias the array in such a way that a variable number, from one to ten, of individual junctions, participated in the locked state. The most important features of his measurements are: a) the locking range in bias current varies linearly with the number of participating junctions; b) the power of the radiation emitted by the array varies with the square of the number of participating junctions.

Work on the theoretical interpretation of the experiment is in progress 40 .

It should be mentioned that recently there has been considerable progress also with coupled (very) small area junctions. Although the coupling circuitry tends to be complicated and fabrication involves E-beam 1 ithography, the recent results 41 are very promising. A 40 junction array delivered up to $1 \mu \mathrm{W}$ of power into a $60 \Omega$ on chip detector at frequencies in the range $350-380 \mathrm{GHz}$; the potential for yet higher powers and higher frequencies exists also in this technology. No measurement of the linewidth were reported, but it is believed to be substantially higher than the $\mathrm{kHz}$ linewidth observed for the soliton oscillators.

\subsection{Flux Flow Oscillators.}

A device that is somewhat similar to the single long Josephson soliton oscillator is the socalled flux flow oscillator developed and described in a serie of important papers by the group at Kyushu University42-45. In the flux flow oscillator a magnetic field is applied to the long Josephson junction, where it breaks the almost symmetric, resonant configuration considered above. Fluxons are created in one end of the junction and annihilated in the other end, i.e. transferred to an external circuit. With a sufficiently large magnetic field, the pulse character of the individual fluxons is changed to an overlapping train of fluxons propagating in one direction. The results that have been obtained are impressive 42-45. In a low impedance receiver on the same chip was measured about $1 \mu \mathrm{W}$ of power tunable between $100 \mathrm{GHz}$ and $500 \mathrm{GHz}$ by means of a magnetic field. No direct experimental measurement of the linewidth has been obtained, however, in an estimate based on the dynamic resistance of the IV curve predicts a linewidth of order some tens of $\mathrm{kHz}^{44}$.

In summary the flux flow oscillator seems to be very we 11 developed by the Japanese group and should be mature for applications.

\section{Analog Amplifiers.}

The Josephson junction has demonstrated superior properties in almost all areas of electronics. It is therefore remarkable that the fundamental element - a Josephson transistor - does not directly exist. However, Likharev et al. 46 suggested that an overlap JTL with current injection in many points in parallel is almost a complete analog of a semiconductor transistor, where the role of electric charge carriers is being played by fluxons. A somewhat similar concept was investigated experimentally by van Zeghbroeck 47 who found a current gain of order 2, very fast response and low power dissipation. 


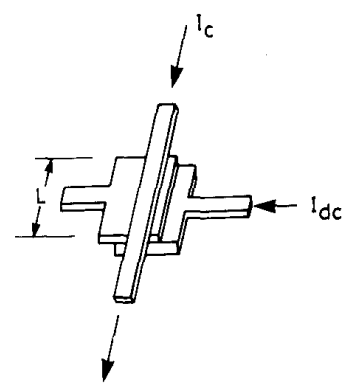

Fig. 8 Schematic diagram of the vortex flow transistor.

The most investigated device experimentally is the socalled vortex flow transistor (VFT) schematically shown in Fig. 8. It consists of one or two long Josephson junctions, where the control current is applied either as a direct current injection or as a magnetic field produced by the current in the control line. When the magnetic field thus produced exceeds the critical magnetic field, a vortex train is produced. These fluxons are set in motion by the external transport current.

The voltage state due to the motion of the fluxon is sensitive to the control current (or magnetic field) and the operating point will move along the load line much as in a transistor. This vortex flow transistor is a very close dual to the semiconductor field effect transistor (FET). The FET is in its simplest form a high impedance voltage controlled current source. The VFT is more like a low impedance current controlled voltage source. Likewise the role of charge carriers (electrons) in the FET is replaced by fluxons in the VFT.

The first experimental version was reported by Rajevakumar ${ }^{48}$ followed by improved versions by Nagatsuma et al. 49 , Mc Ginnis et a1.50 and Hashimoto et a1.51. Very remarkably, a device with 50 VFT have predicted usefuT gains of order $10-20 \mathrm{~dB}$ up to $100 \mathrm{GHz}^{52}$, al though this has not been confirmed in the experiments 50 .

\section{Other Applications.}

The beautiful and intriguing nature of the fluxons on the Josephson transmission line has led researchers to imagine other applications that have not been discussed above and some of which have not been pursued experimentally yet. One such example is to use the gyroscopic effects in a rotating ring with fluxons 53 . According to the suggestion this could be used as a rotation rate sensor. In an analog application of the flux shuttle, a current amplifier and a D to A converter can be constructed 54 . Work with JTL superlattices 55 and JTL's with spatially periodic perturbations 56 that is going on presently may also lead to new applications. Space limitations, however, does not allow us to discuss that here.

\section{Summary.}

Fluxons on Josephson transmission lines have many surprising and usefur properties as I hope to have demonstrated in this review. As in many other branches of of superconducting electronics the research is still going on and every year brings new and surprising results, that may soon lead to useful applications.

\section{Acknowledgements.}

S. Sakai is aknowledged for his valuable comment during the preparation of the manuscript.

\section{References}

1. A. Barone and G. Paterno: "Physics and Applications of the Josephson Effect". Wiley, New York, 1982)

2. N.F. Pedersen, Chapter 9 in "Solitons". Eds. S.E. Trullinger, V.E. Zakharov and V.L. Pokrovsky (Elsevier, 1986)

3. D.W. McLaughl in and A.C. Scott, Phys. Rev. A 18, 1652 (1978)

4. P.M. Marcus and Y. Imry, Sol.St.Comm. 33, 345 (1980)

5. M. Buttiker and H. Thomas, Phys.Rev. A 37, 235 (1988)

6. N.F. Pedersen, M.R. Samuelsen and D. Welner, Phys Rev. B 30, 4057 (1984)

7. A. Davidson and N.F. Pedersen, unpublished

8. S. Hontsu and J. Ishii, J.Appl. Phys.63, 2021 (1988)

9. A.R. Bishop. B. Horowitz and P.S. Lomdahl, Phys.Rev. B 38, 4853 (1988)

10. M. Cirillo, R.D. Parmentier and B. Savo, Physica 3D, 565 (1981)

11. A. Davidson, B. Dueholm and N.F. Pedersen, J.Appl. Phys. 60, 1447 (1986)

12. T.A. Fulton and R.C. Dynes, Sol.St. Comm. 12, 57 (1972)

13. J. Nitta and A. Matsuda, Phys.Rev. B 35, 4764 (1987)

14. H. Akoh, S. Sakai and S. Takada, Phys.Rev. B 35, 5357 (1987)

15. A. Fujimaki, K. Nakajima and Y. Sawada, Phys. Rev. Lett. 59, 2895 (1987)

16. K. Nakajima, H. Mizusawa, H. Akoh, S. Takada and Y. Sawada, preprint (1990)

17. H.F. Hess et a1. , Phys.Rev. Lett. 62, 214 (1989)

18. T. Matsuda et a1., Phys. Rev. Lett. 62, 2519 (1989)

19. N.F. Pedersen and D. Welner, Phys. Rev. B 29, 2551 (1984)

20. B. Dueholm, O.A. Levring, J. Mygind, N.F. Pedersen, 0.H. Soerensen, and M. Cirillo, Phys. Rev. Lett. 46, 1299 (1981).

21. H. Akoh, S. Sakai, A. Yagi, and H. Hayakawa, IEEE, Trans. on Magn. MAG-21,

p. 737 (1985)

22. T.A. Fulton, R.C. Dynes and P.W. Anderson, Proc. IEEE 61, 28 (1973)

23. T.A. Fulton and L.N. Dunkleberger, Appl. Phys. Lett., vol. 22, p. 232 1973) 
3334

24. K. Nakajima, Y. Onodera, and Y. Ogawa, Appl Phys., p. 1620 (1976)

25. T.V. Rajeevakumar, IEEE Trans.Magn. MAG-17, 591 (1981)

26. S. Sakai, H. Akoh, and H. Hayakawa, Extended Abstracts of 5 th Inter. Conf. on Solid State Devices and Materials, (Kobe, 1984) p. 631; Jpn. J. Appl. Phys. 24 L771 (1985) 27. S. Sakai, H. Akoh, and H. Hayakawa, Jpn. J. Appl. Phys. 24 L749 (1985)

28. S. Sakai, H. Akoh, and S. Takada, extended abstracts of the ISEC'87 conference; Tokyo 1987, p. 118

29. S. Sakai, Bullet in of The Electrotechnical Laboratory, vol. 53, 901 (1989)

30. K. Nakajima, H. Sugahara, A. Fujimaki, and Y. Sawada, App1. Phys. 66, 949 (1989)

31. E. Joergensen, V.P. Koshelets, R. Monaco, J.

Mygind, M.R.Samuelsen, and M. Salerno,

Phys.Rev.Lett.49, 1093 (1982)

32. G. Hohenwarter, Private communication

33. N. Gronbech-Jensen, N.F. Pedersen, A.

Davidson, and R.D.Parmentier, Phys. Rev. B (in print)

34. M. Cirillo, I. Modena, P. Carelli, and V. Foglietti, J. App 1. Phys. $\underline{65}, 2376$ (1989)

35. S. Sakai, H. Akoh, and H. Hayakawa, Jpn J.Appl.Phys.23, L610(1984)

36. S. Pagano, R. Monaco, and G. Costabile, IEEE Trans. Magn. MAG-25 1080 (1989)

37. R. Monaco, S. Pagano and G. Costabile, Phys.Lett. A131, 122(1988)

38. T. Holst, J. Bindslev Hansen, N. Gronbech-Jensen, and I.A. B1ackburn. Phys.Rev. B42, 127 (1990)

39. R. Monaco, private communication

40. R. Monaco, R.D. Parmentier, and N. Gronbech-Jensen, to be published

41. K. Wain, A.K. Jain and J.E. Lukens, App1. Phys. Letters 54,1805 (1989)

42. T. Nagatsuma, K. Enpuku, F. Ine, and K. Yoshida, J.App 1.Phys.54, 3302(1983)

43. T. Nagatsuma, K. Enpuku, K. Yoshida, and F. Irie, J.App1.Phys. 56, 3284 (1984)

44. T. Nagatsuma, K. Enpuku, K. Sueoka, K. Yoshida, and F. Irie, J.App1.Phys. 58, 441(1985

45. J. Qin, K. Enpuku, and K. Yoshida, J.Appl.Phys.63, $1130(1988)$

46. K.K. Likharev, V.K. Semenov, 0.V. Snigirev, and B.N. Todorov, IEEE Trans.Magn. MAG-15, 420 (1979)

47. B.J. van Zeghbroeck, IEEE Trans. Magn. MAG-21 916 (1985)

48. T.J. Rajeevakumar, App. Phys. Lett. 39, 439 (1981)

49. T. Nagatsuma, K. Enpuku, H. Iwakura and K. Yoshida, Jap. Journ. of App. Phys. 24, 599 (1985)

50. D.P. McGinnis, G.K. Hohenwarter, M. Ketkar, J.B. Beyer, and J.E. Nordman, IEEE Trans. Mag. MAG-25, 1258 (1989)

51. T. Hashimoto, K. Enpuku and K. Yoshida IEEE Trans. Magn. MAG-25, 1266 (1989)
52. D.P. McGinnis, J.B. Beyer and J.E. Nordman, J. App1. Phys. 59. 3917 (1986)

53. F. Marchesoni, App1. Phys. Lett. 49, 1115 (1986)

54. M.F. Sweeny, Unpublished manuscript (1984)

55. D. Barday and M. Remoissenet Phys, Rev, B41, 10387 (1990) 56. B.A. Malomed and A.V. Ustinov, Phys. Rev. B41, 254 (1990) 